\title{
Assigning confidence scores to homoeologs using fuzzy logic
}

\author{
Natasha M Glover Corresp., 1, 2,3 $^{\text {, Adrian Altenhoff }}{ }^{1,4}$, Christophe Dessimoz ${ }^{1,2,3,5,6}$ \\ ${ }^{1}$ SIB Swiss Institute of Bioinformatics, Lausanne, Switzerland \\ 2 \\ 3 Department of Computational Biology, University of Lausanne, Lausanne, Switzerland \\ 4 Department of Computer Science, ETH Zurich, Zurich, Switzerland \\ 5 Department of Genetics, Evolution, and Environment, University College London, London, United Kingdom \\ 6 Department of Computer Science, University College London, London, United Kingdom \\ Corresponding Author: Natasha M Glover \\ Email address: natasha.glover@unil.ch
}

In polyploid genomes, homoeologs are a specific subtype of homologs, and can be thought of as orthologs between subgenomes. In Orthologous MAtrix (OMA), we infer homoeologs in three polyploid plant species: upland cotton (Gossypium hirsutum), rapeseed (Brassica napus), and bread wheat (Triticum aestivum). While we can typically recognize the features of a "good" homoeolog prediction (a consistent evolutionary distance, high synteny, and a one-to-one relationship), none of them is a hardfast criterion.

We devised a novel fuzzy logic-based method to assign confidence scores to each pair of predicted homoeologs. We inferred homoeolog pairs and used the new and improved method to assign confidence scores, which ranged from 0-100. Most confidence scores were between 70-100, but the distribution varied between genomes. The new confidence scores show an improvement over our previous method and were manually evaluated using a subset from various confidence ranges. 


\section{Assigning Confidence Scores to Homoeologs using Fuzzy Logic}

2 Natasha M. Glover ${ }^{1,2,3}$, Adrian Altenhoff ${ }^{1,4}$, Christophe Dessimoz ${ }^{1,2,3,5,6}$

3

4 1. SIB Swiss Institute of Bioinformatics, 1015 Lausanne, Switzerland

5 2. Center for Integrative Genomics, University of Lausanne, 1015 Lausanne, Switzerland

6 3. Dept. of Computational Biology, University of Lausanne, 1015 Lausanne, Switzerland

7 4. ETH Zurich, Computer Science, Universitätstrasse 6, 8092 Zurich, Switzerland

8 5. Dept. of Genetics, Evolution \& Environment, University College London, Gower St,

6. Dept. of Computer Science, University College London, Gower St, London WC1E 6BT,

Corresponding author: Natasha Glover ${ }^{1,2,3}$

14 Email address: natasha.glover@unil.ch 


\section{ABSTRACT}

In polyploid genomes, homoeologs are a specific subtype of homologs, and can be thought of as orthologs between subgenomes. In Orthologous MAtrix (OMA), we infer homoeologs in three polyploid plant species: upland cotton (Gossypium hirsutum), rapeseed (Brassica napus), and bread wheat (Triticum aestivum). While we can typically recognize the features of a "good" homoeolog prediction (a consistent evolutionary distance, high synteny, and a one-to-one relationship), none of them is a hard-fast criterion.

We devised a novel fuzzy logic-based method to assign confidence scores to each pair of predicted homoeologs. We inferred homoeolog pairs and used the new and improved method to assign confidence scores, which ranged from 0-100. Most confidence scores were between 70100 , but the distribution varied between genomes. The new confidence scores show an improvement over our previous method and were manually evaluated using a subset from various confidence ranges.

\section{INTRODUCTION}

Polyploidy is an important and widespread phenomenon within the plant kingdom (De Bodt, Maere \& Van de Peer, 2005), and redundancy at the level of chromosomes implies redundancy at the level of genes. More specifically, homoeologs are defined as genes which originated by a speciation event, diverged, and came back together some time later via allopolyploidization (Glover, Redestig \& Dessimoz, 2016). (See Table 1 for definitions of terms related to polyploidy.) The homoeologous relationships between subgenomes of a polyploid can be used in order to determine the structural, genetic, and evolutionary results of polyploidization.

Since 2015 we have included pairwise homoeolog predictions between subgenomes in OMA (Orthologous MAtrix), which is a method and database for inferring evolutionary relationships (Altenhoff et al., 2015). With other methods, homoeologs are usually predicted using a best bidirectional hit approach, sometimes in combination with an added requirement of positional conservation, i.e. synteny (Dewey, 2011). However, one-to-one correspondence or synteny among homoeologs may not hold for several reasons, such as lineage-specific duplications or 
46 small-scale translocations. In OMA, we do not rely on synteny for homoeolog inference, and we

47 also allow for duplications after the hybridization event.

48 However, relaxing one-to-one and synteny criteria makes it harder to distinguish correct from 49 incorrect calls. Furthermore, because of the redundancy and size, polyploid genomes can be 50 difficult to assemble and annotate. For instance, Triticum aestivum (bread wheat) remains in a 51 highly fragmented survey state, consisting of scaffolds rather than fully-assembled chromosomes 52 (International Wheat Genome Sequencing Consortium (IWGSC), 2014; Clavijo et al., 2017). 53 This previously motivated us to classify homoeolog predictions as "high" versus "low" 54 confidence based on chromosome matching (global synteny) (Altenhoff et al., 2015). For 55 example, if one homoeolog of a pair was on chromosome 3B while the other homoeolog is on 56 chromosome 3A, this pair was considered high confidence because they belong to the 57 chromosome group 3. While this holds true most of the time due to the relatively short 58 divergence time between subgenomes, this is coarse, and can be misleading in the presence of 59 chromosomal rearrangements or small-scale translocations.

60 There have been several methods reported which yield quantitative confidence scores for 61 ortholog or paralog predictions. For example, InParanoid assigns confidence scores to in62 paralogs on a scale from 0 to 100 depending on how distant the predicted inparalog sequence is 63 from the 'main' ortholog. Additionally, InParanoid confidence scores are assigned to 64 orthologous groups based on a technique that assigns a higher score to potential ortholog 65 sequences that have much better bootstrap value than competing ortholog sequences (Remm, 66 Storm \& Sonnhammer, 2001). Several meta-methods for predicting orthology (i.e. those that 67 combine the results of many different orthology inference algorithms) report confidence scores. 68 For example, Metaphors gives confidences scores based on the number of independent sources 69 the orthology prediction was found in, as well as scores based on the consistency for which 70 scores are computed using their method (Pryszcz, Huerta-Cepas \& Gabaldón, 2011). The 71 Drosophila RNAi Screening Center Integrative Ortholog Prediction Tool (DIOPT) gives 72 confidence scores based on not only the number of independent algorithms predicting each pair, 73 but also weighted to reflect the functional similarity, as shown by GO semantic similarity (Hu et 74 al., 2011). A recent method called WORMHOLE uses 17 different ortholog prediction tools in a 75 supervised machine learning algorithm for predicting least diverged orthologs (Sutphin et al., 
76 2016). The confidence scores reported are based on the number of algorithms predicting each 77 pair, as well as the support vectors machine classifiers of the WORMHOLE algorithm — both 78 scaled between 0 and 1 with 0.5 being the best precision-recall balance.

79 To our knowledge, there have not been any quantitative confidence scores of homoeolog 80 predictions reported. However, there has been qualitative confidence reported for some 81 polyploids. In a paper by Cheng et al., in the triploid Brassica rapa species, they gave qualitative 82 confidence scores (high vs. low) for homoeolog pairs. Pairs were determined as high confidence 83 if the gene was supported by transcriptome evidence, and if there was a syntenic ortholog in 84 Arabidopsis (Cheng et al 2012).

85 Here, we introduce a more fine-grained and flexible confidence score for homoeolog predictions.

86 87 88 89 90

Based on fuzzy logic, it combines evolutionary distance, local synteny, and the extent of duplication. Fuzzy logic is about "degrees of truth" rather than binary true or false and is based on the idea that how true or not something is can be represented over a continuum. (See Table 2 for terminology related to fuzzy logic.) Most existing applications of fuzzy logic deal with control systems (Cheng \& Yeh, 1993; Hirulkar et al., 2014). In our case, we can recognize homoeologs which are most certainly true predictions; we can also recognize homoeologs which are almost certainly wrong. However, the homoeolog predictions which are more dubious are harder to assign a score to. Fuzzy logic resembles human reasoning because it works on a range of possibilities for input, expressed in linguistic terms. This is useful for practical purposes- it helps deal with uncertainty, when the lines between what is a good and bad homoeolog prediction are fuzzy.

\section{MATERIALS AND METHODS}

In the latest OMA release, we include three agriculturally important allopolyploid crops: Triticum aestivum (bread wheat), Brassica napus (rapeseed) and Gossypium hirsutum (upland cotton). We used these genomes to infer homoeologous pairs of genes, with the improved confidence score assignment. We then evaluated our homoeolog predictions by comparing the confidence scores to other data aggregated from OMA, and by manually assessing a subset of the predictions. 
105

106 107 108

109

110

111

112

113

114

115

116

117

118

119

120

121

122

123

124

125

126

127

128

129

130

131

132

133

\section{Polyploid genomes used}

Upland cotton (Gossypium hirsutum; $\mathrm{A}_{t} \mathrm{~A}_{t} \mathrm{D}_{t} \mathrm{D}_{\mathrm{t}} ; 2 \mathrm{n}=4 \mathrm{x}=52$ ) is an important fiber crop, making up 90\% of cotton production worldwide (Zhang et al., 2015). The diploid Gossypium progenitors diverged 5-10 MYA, hybridized 1-2 MYA, and was followed by a genome doubling, giving the allotetraploid cotton (Wendel \& Cronn, 2003). The genome size of this allotetraploid is 2.25 Gb, assembled into 26 chromosomes. The "TM-1" G. hirsutum genome, NAU-NBI assembly and annotation v1.1 (Zhang et al., 2015), was obtained from ftp://ftp.bioinfo.wsu.edu/species/Gossypium_hirsutum/NAUNBI_G.hirsutum_AD1genome/genes/. A total of 66967 protein-coding genes were used for homoeolog inference.

Oilseed rape (Brassica napus; $2 \mathrm{n}=4 \mathrm{x}=38$ ) is an allotetraploid member of the Brassicaceae (mustard/cabbage) family. The progenitors of Brassica napus diverged about 4 MYA, followed by a relatively recent hybridization, dating back to 7,500-12,500 years ago (Chalhoub et al., 2014). The $1.13 \mathrm{~Gb}$ Brassica napus genome was sequenced and assembled into 19 pseudomolecules, with 98130 canonical protein coding genes (Chalhoub et al., 2014). The "Darmor-bzh v5" version of the B. napus genome was obtained from (http://www.genoscope.cns.fr/brassicanapus/data/).

Wheat (Triticum aestivum) is a staple food. Its genome is large ( $\sim 17 \mathrm{~Gb})$ and allohexaploid, consisting of 3 subgenomes with 7 sets of chromosomes each $(6 x=2 n=42)$. The divergence of the progenitor species is estimated to be around 7 MYA, and the two hybridization events $<1$ MYA (International Wheat Genome Sequencing Consortium (IWGSC), 2014). The wheat genome was obtained from Ensembl Plants 33 using the TGACv1 assembly (Clavijo et al., 2017) of the “Chinese Spring” cultivar; ftp://ftp.ensembl.org/pub/release-33/embl/triticum_aestivum/. The annotation consisted of 103458 genes after removing alternative splice variants. Canonical genes among the splice variants were chosen based on the method described in (Altenhoff et al., 2011).

\section{Homoeolog inference}

In OMA, we inferred homoeologs in the polyploid species by treating each subgenome as a separate genome and inferring orthologs following the normal OMA pipeline (Train et al., 2017). Briefly, the steps for obtaining pairwise homoeologs are as follows: 
134

135

136

137

138

139

140

141

142

143

144

145

146

147

148

149

150

151

152

153

154

155

156

157

158

159

160

161

- Subgenome delineation: Polyploid genomes were separated into two or more subgenomes based on their identifiers. From here on, a subgenome is treated as a standalone genome/proteome.

- Homology inference: Using proteomes from all the species in OMA, Smith-Waterman alignments were made with all possible pairs of sequences from all genomes (Smith \& Waterman, 1981). Pairs of protein sequences from different genomes (or subgenomes) with sufficient alignment score and overlap were retained.

- Homoeolog and co-homoeolog inference: Pairs retained from the previous step that are the mutually evolutionary closest sequences between a pair of subgenomes are kept during this step. In order to include many-to-many homoeologous relationships, pairs found within a confidence interval of the mutually closest sequences are kept.

- Witness of "non-homoeology" verification: In order to avoid paralogs to be mistakenly identified as homoeologs due to differential gene loss (Dessimoz et al., 2006), a verification step is performed by searching for a third genome that retained both orthologous copies. The third genome can be any of the 2103 eukaryotic, prokaryotic, or archaeal genomes currently in OMA, but are often very closely related species. This third genome acts as a witnesses of non-homoeology, and pairs that do not pass this test are filtered out.

Recently, several improvements to the OMA algorithm were introduced (Train et al., 2017). This included refinements to account for rapidly evolving duplicated genes, as well as fragmentary sequences. Additionally, those homoeologs which were considered outliers in the previous OMA algorithm (Altenhoff et al., 2015) are now retained.

All data, including the genome information and homoeologous/orthologous relationships, is stored in an HDF5 database (https://omabrowser.org/All.Dec2017/OmaServer.h5), and queried programmatically using python. Our code for the confidence score computations can be found in Supplemental File 1. Skfuzzy (https://github.com/scikit-fuzzy/scikit-fuzzy) was used for all functions related to fuzzy logic. Only canonical genes were considered (no alternative splice variants) for the following analyses. 
162

163 164

165 166

167

169

170

171

172

173

174

175

176

177

178

179

180

181

182

183

184

185

186

187

188

189

\section{Variables used as input for fuzzy sets}

Three inputs, i.e. features of a given homoeolog pair, were used for the fuzzy logic variables: the evolutionary distance, synteny score, and total copy number.

Synteny is the overall conservation of chromosome order and location of genes when comparing two chromosomes. However, rearrangements may result in smaller regions of the chromosome being syntenic, rather than the whole chromosome. Thus, we computed a local synteny score for each pair of homoeologs (Figure 1). This consisted of obtaining two windows of genes, one on each subgenome: the first one containing the 10 neighbor genes surrounding one homoeolog, and the second window containing the 10 neighbor genes surrounding the corresponding homoeolog. If the homoeolog was on a scaffold with less than 10 genes, all the genes on the scaffold were used. However, if there were less than two genes (including the homoeolog) on the scaffold, we assigned the synteny score to 0 for these pairs. We then computed the proportion of genes (not including the homoeologs) in each window which were homoeologous to the corresponding window on the other subgenome and took the mean of these two as the synteny score.

The evolutionary distance is based on the number of nucleotide substitutions between two sequences. This is in PAM units, and calculated as part of the normal OMA algorithm (Roth, Gonnet \& Dessimoz, 2008). A distance of 1 PAM unit describes an amount of evolution which will change, on average, $1 \%$ of the amino acids.

The "total copy number" is a metric to understand the degree of duplication for a pair of homoeologs. For a given pair, it is calculated as the number of homoeologs for the first gene + the number of homoeologs for the second gene.

\section{Defining the universe of discourse and membership functions for all variables}

For each genome, input variable (synteny, evolutionary distance, total copy nr), and output variable (confidence score), the universe of discourse is the range of possible values. The universe for each variable was are defined in Table 3, using the "Antecedent" and "Consequent" functions from the skfuzzy control module. Gaussian curve membership functions were defined for each genome using the skfuzzy "gaussmf" function. The membership classes for each fuzzy set are defined in Table 4. 
191 We created five rules based on the universes defined above and and stored them in the lookup 192 table (see Results). The control system and simulation were made using the skfuzzy control 193 module and the rules. This simulation was then used to defuzzify, i.e. return a crisp output, using 194 the centroid defuzzify method. It takes the inputs and returns a confidence score between 0-100. 195 We then kept the smallest confidence score returned as the minimum and scaled the maximum 196 confidence score to be 100 . A set of 30 homoeolog pairs were manually evaluated in $G$. 197 hirsutum. Ten pairs were randomly chosen from the 0-60, 60-90, and 90-100 confidence score 198 ranges. Putative functions were found by searching the protein sequence in the NCBI Conserved 199 Domain Database (CDD) (Marchler-Bauer et al., 2017).

\section{RESULTS}

201 Polyploid species and homoeolog inference

202 We inferred homoeologs for three polyploid species using OMA: Gossypium hirsutum (GOSHI), 203 Brassica napus (BRANA), and Triticum aestivum (WHEAT). In the OMA pipeline, 204 homoeologs are predicted as orthologs between subgenomes, thus, the genes have to be 205 annotated as belonging to a particular subgenome in order to infer homoeology. However, as 206 assemblies can be in different states of draft, not all genes are always mapped to a specific 207 chromosome or subgenome. We therefore discarded 2002, 515, and 4151 genes from GOSHI, 208 BRANA, and WHEAT respectively as they were not previously mapped to any particular 209 subgenome. This left between $96-99.5 \%$ of the total genes in each genome to infer homoeology. 210 Using these genes, we predicted pairs of homoeologs between subgenomes for each of the three 211 species. This resulted in 30230, 35661, and 88513 pairs of homoeologs for GOSHI, BRANA, 212 and WHEAT, respectively.

\section{Investigation into global versus local synteny}

214 Rearrangements may result in smaller regions of the chromosome being syntenic, rather than the 215 whole chromosome. In order to justify using a local synteny score rather than a global synteny

216 based on chromosome matching between subgenomes, we computed the number of homoeologs 
217 across pairs of chromosomes between two subgenomes in each species. With OMA we predicted 218 many homoeologs across different chromosome groups (Figure 2).

219 Non-homoeologous chromosomes, i.e. different chromosome groups, with increased frequency 220 of homoeologs are consistent with known reciprocal translocations. For example, in GOSHI, 221 there are two known large reciprocal translocations: parts of the chromosomes were exchanged 222 between $\mathrm{A} 02$ and $\mathrm{A} 03$, as well as between A04 and A05. We would be able to see this by an 223 increased frequency of homoeolog pairs inferred between chromosomes not belonging to the 224 same chromosome group (Figure 3). Indeed, with OMA we inferred 692, 1208, 598, and 696 225 pairs of homoeologs between chromosomes A02/D03, A03/D02, A04/D05, and A05/D04, 226 respectively (Figure 2). This is significantly higher than the mean number of homoeolog pairs for 227 non-homoeologous chromosomes. We observe even more levels of chromosomal translocation 228 in WHEAT and BRANA as well, for example detecting the known reciprocal translocations in 229 WHEAT between 4A, 5A, and 7B (Ma et al., 2013).

230 Additionally, chromosome pairs with a few number of homoeologs may represent single-gene 231 translocations between non-homoeologous chromosomes and should not be discarded from 232 homoeology prediction via synteny-based methods. Taken together, these results suggest that a 233 local synteny score is more robust than global synteny in order to account for large and small 234 translocations.

Description of Fuzzy Logic Inputs

236 For each homoeolog pair, we used the synteny score, the evolutionary distance, and the total 237 copy number as input.

238 The synteny score is the degree of local gene neighborhood conservation. Although synteny is 239 not a hard requirement for homeologs, a conservation of synteny is a good indicator of correct 240 homoeolog predictions. In order to account for chromosomal rearrangements, as well as genome 241 assemblies which are not yet fully assembled into pseudomolecules, we computed a local 242 synteny score. This technique, however, only works when both genes of a homoeolog pair have 243 at least one neighbor gene. Therefore, we could not compute the synteny score for homoeologs 244 that were on small scaffolds with only one gene annotated. For those pairs we set the synteny 245 score to 0 . This was 490, 0, and 36250 pairs for GOSHI, BRANA, and WHEAT, respectively. 
246 The evolutionary distance is based on the number of nucleotide substitutions between two 247 sequences (in PAM units). Because of the relatively short divergence between subgenomes, we 248 expect there to generally be a low distance between homoeologs. Additionally, genes which have 249 a high number of predicted homoeologs could indicate something suspect, such as a transposable 250 element misannoted as a gene. All distributions of the inputs are shown in Figure 4.

251 Fuzzification of data into membership functions

252 The membership functions allow us to translate an input value into a degree of membership 253 between 0 and 1 . The membership curves are overlapping to account for the fuzziness between 254 categories, and for all genomes we defined what we consider to be low, medium, or high input 255 values (Figure 5). For example, a homoeolog pair with a synteny score of 0.6 could then be 256 translated linguistically to not at all low synteny, somewhat medium synteny, and mostly high 257 synteny. For synteny, the curves are statically defined based on the synteny input universe 258 (possible scores only from 0 to 1), while the distance and total copy number are based on the 259 minimum, maximum, and median values for that particular genome (see Materials and Methods).

\section{The Fuzzy Rules and the control output}

261 The output from our fuzzy inference process, Confidence, also has a membership function. It is 262 used to map the fuzzy confidence to a crisp confidence score, between 0 and 100 (Figure 6).

263 Fuzzy logic uses a set of IF THEN statements (rules) for mapping the input space to an output 264 space. We set the rules as to what we think should determine the five categories of confidence 265 (Figure 6). The very high confidence homoeologs are those which have a high synteny, a low 266 distance, and a low total number of homoeologs. The very low confidence homoeologs have a 267 low synteny score, a high distance, and a high copy number of homoeologs. High, medium, and 268 low confidence is everything in between, based on what we would consider low or high inputs. 269 We put a lot of emphasis on synteny score because the chances of a non-homoeologous pair 270 being syntenic but not originating from a common ancestral gene is very low.

\section{Defuzzification}

272 After defining the rules, we created a control system and simulation for each of the genomes.

273 The inputs for each homoeolog pair were then fed into the simulation which contains the rules, 
274 and defuzzified. The defuzzification process converts the fuzzy linguistic confidence to a crisp 275 confidence score, which we then scaled between the minimum value and 100. The reason for 276 scaling to 100 is so that there would not be a sharp cutoff and a maximum confidence score 277 around 80. This facilitates comparison of homoeolog confidence scores within genomes, as 278 people naturally tend to associate the best score with 100 . The resulting distribution of 279 confidence scores is shown in Figure 7. Unscaled confidence scores are shown in Supplementary 280 Figure 1.

281

282 283 284

285 286

287 288 289

290

291

292

293

294

295

296

297

298

299

300 301 302

\section{Evaluation of homoeologs confidence scores}

We assessed the homoeolog predictions by looking at the correlation between the total number of orthologs per homoeolog pair and the confidence score. We also manually evaluated a set of 10 homoeolog pairs from 0-60, 60-90, and 90-100 confidence score ranges (Supplemental Table 1).

The total number of orthologs takes into account the ortholog predictions for all of the species in OMA. Homoeolog pairs with few orthologs are either lineage-specific or dubious, whereas pairs with many orthologs represent those more likely to be true. Although the correlation is low between the total number of orthologs and the confidence score $(\mathrm{R}=0.005)$, it holds as a general trend when looking at confidence scores between 0-70 versus confidence scores between 70-100 (Figure 8). In the set of GOSHI homoeologs manually evaluated, the total number of orthologs was significantly lower for the low confidence homoeologs (0-60), than the 60-90 and 90-100 samples (Supp. Table 1).

Interestingly, in GOSHI, for the set of 10 manually evaluated pairs from 0-60 confidence, half had RVT-3 (reverse transcriptase-like) domains. According to the CDD description, "This domain is found in plants and appears to be part of a retrotransposon." This could explain the few number of orthologs for those with low confidence scores, because transposable elements (TE) rapidly evolve and may have lost homology in the other species. By contrast, none of the sampled homoeologs with a confidence score above 60 had RVT-3 annotation, or any functional description associated with TEs.

Finally, in order to compare our new confidence scores to the previous confidence classes in OMA, we looked at the proportion of pairs in each confidence score bin which were previously marked as either high or low confidence (Figure 9). There are a significant amount of homoeolog 
303 pairs which were previously classified as low confidence, but are now between 90-100 304 confidence score $(37 \%, 31 \%, 5 \%$ of the formerly low confidence pairs in GOSHI, BRANA, and

305 WHEAT respectively). On the other hand, there was a much smaller proportion of pairs that

306

307

308

309

310

311

312 DISCUSSION

313 Fuzzy logic has some applications in biology (Torres \& Nieto, 2006; Chandgude \& Pawar, 314 2016), but none in the field of homology prediction or for assigning confidence scores for 315 were considered high confidence before, but now have less than 70 as a confidence score $(0.5 \%$, $1 \%$, and $4 \%$ for GOSHI, BRANA, and WHEAT, respectively). In wheat, the large peak of scores between $70-80$ that were formerly high confidence is because $68 \%$ of these pairs are on small scaffolds which we were unable to compute synteny for, however they belong to the same chromosome group. inferences for which a ground-truth cannot be established. Our application therefore describes an interesting and novel approach to deal with such scenarios where supervised machine learning approaches are risky to be applied.

Although our methods of defining membership functions and rules for the confidence scores may seem ad hoc, that is where fuzzy logic excels. We don't claim this method to be objective or the best schema, however after manual evaluation, the results are interpretable and useful. An important limitation to our approach is that the confidence scores are heavily based on synteny, which is known to degrade over evolutionary time. Therefore, synteny may be low or even undetectable for older polyploids. The species used in this study are relatively young polyploids, and this approach was untested in paleopolyploids. Nevertheless, the results are relevant for the three polyploid species in OMA, and are reproducible as well, as they are coded into the OMA pipeline.

Synteny has been used already as a way to assess the confidence of ortholog pairs. For example, in Ensembl, a "gene order conservation" score uses a window of two genes on each side of a given ortholog prediction and checks whether the genes are also orthologs and in the same orientation. Furthermore, they calculate a "whole genome alignment" score which assesses the proportion of a given ortholog pair which fall within syntenic regions, with more weight given to 
332 exons that can be aligned than introns (Aken et al 2017). In the SYNERGY algorithm, Wapinksi

333 et al (2007) used the synteny combined with the evolutionary distance to make the ortholog

334 predictions themselves rather than to assess ortholog predictions.

335 The new homoeolog confidence scores are an improvement to the old way we assigned

336 confidence class. Going from a discrete category of high vs. low to a quantitative score can give

337 users a wider range of options depending on the analyses they want to perform. For example, for

338 finding differential gene expression among homoeologs, one may want to be conservative and

339 take on those pairs with $90+$ confidence. On the other hand, if scanning for all potential

340 homoeologs that could provide disease resistance, some potential R-genes may look like highly

341 repetitive TEs (Bayer, Edwards \& Batley, 2018). With our fuzzy logic approach, the homoeolog

342 pairs would have a low confidence score due to their high Total Copy Number. Thus, one could

343 take all of the homoeologs no matter the confidence.

344 Between the polyploid species used in this study, there are genome specificities, biological as

345 well as assembly-wise, which is why we see differences in terms of confidence scores. For

346 example, wheat has the majority of its assembly still in scaffolds, which is why the peak of

347 scores is around 70-80. These confidence scores will most likely increase when the assembly

348 improves. However, by using the local synteny, our method at least allows us to make a

349 confidence score using scaffolds.

350 Homoeologs aren't always syntenic, in one-to-one copies, or with a low distance. Pairs on non-

351 matching chromosomes may be homoeologs that represent single-gene translocations.

352 Furthermore, some genes can evolve quickly, giving an abnormally high distance. Additionally,

353 some genes might have a high copy number. These could be real genes that have a propensity

354 for duplication (depends on the function, located in a recombination hotspot, gene balance

355 hypothesis, etc). It is important to not disregard these pairs in homoeolog inference methodology,

356 as they could still represent interesting functions.

\section{CONCLUSION}

358 To assign confidence scores to inferred pairs of homoeologs, we introduced a fuzzy logic-based 359 method combining evolutionary distance, local synteny, and cardinality of homoeology

360 relationships. Even though there is a degree of subjectivity in defining the fuzzy rules, the 
361 resulting scores proved meaningful in how they correlate with the number of orthologs and in a 362 manual inspection of a random subset of 30 instances. The framework constitutes a substantial 363 improvement over the previous confidence score which was only based on global synteny and 364 had much less granularity.

365

366

\section{REFERENCES}

367 368

369

370

371

372

373

374

375

376

377

378

379

380

381

382

383

Altenhoff AM., Schneider A., Gonnet GH., Dessimoz C. 2011. OMA 2011: orthology inference among 1000 complete genomes. Nucleic acids research 39:D289-94.

Altenhoff AM., Škunca N., Glover N., Train C-M., Sueki A., Piližota I., Gori K., Tomiczek B., Müller S., Redestig H., Gonnet GH., Dessimoz C. 2015. The OMA orthology database in 2015: function predictions, better plant support, synteny view and other improvements. Nucleic acids research 43:D240-9.

Bayer PE., Edwards D., Batley J. 2018. Bias in resistance gene prediction due to repeat masking. Nature Plants 4:762.

Chalhoub B., Denoeud F., Liu S., Parkin IAP., Tang H., Wang X., Chiquet J., Belcram H., Tong C., Samans B., Corréa M., Da Silva C., Just J., Falentin C., Koh CS., Le Clainche I., Bernard M., Bento P., Noel B., Labadie K., Alberti A., Charles M., Arnaud D., Guo H., Daviaud C., Alamery S., Jabbari K., Zhao M., Edger PP., Chelaifa H., Tack D., Lassalle G., Mestiri I., Schnel N., Le Paslier M-C., Fan G., Renault V., Bayer PE., Golicz AA., Manoli S., Lee T-H., Thi VHD., Chalabi S., Hu Q., Fan C., Tollenaere R., Lu Y., Battail C., Shen J., Sidebottom CHD., Wang X., Canaguier A., Chauveau A., Bérard A., Deniot G., Guan M., Liu Z., Sun F., Lim YP., Lyons E., Town CD., Bancroft I., Wang X., Meng J., Ma J., Pires JC., King GJ., Brunel D., Delourme R., Renard M., Aury J-M., Adams KL., Batley J., Snowdon RJ., Tost J., Edwards D., Zhou Y., Hua W., Sharpe AG., Paterson AH., Guan C., 
Wincker P. 2014. Plant genetics. Early allopolyploid evolution in the post-Neolithic Brassica napus oilseed genome. Science 345:950-953.

387 Chandgude N., Pawar S. 2016. Diagnosis of diabetes using Fuzzy inference System. In: 2016 International Conference on Computing Communication Control and automation (ICCUBEA). 1-6.

Cheng FF., Yeh SN. 1993. Application of fuzzy logic in the speed control of AC servo system and an intelligent inverter. IEEE Transactions on Energy Conversion 8:312-318.

Clavijo BJ., Venturini L., Schudoma C., Accinelli GG., Kaithakottil G., Wright J., Borrill P., Kettleborough G., Heavens D., Chapman H., Lipscombe J., Barker T., Lu F-H., McKenzie N., Raats D., Ramirez-Gonzalez RH., Coince A., Peel N., Percival-Alwyn L., Duncan O., Trösch J., Yu G., Bolser DM., Namaati G., Kerhornou A., Spannagl M., Gundlach H., and annotation of the allohexaploid wheat genome identifies complete families of agronomic genes and provides genomic evidence for chromosomal translocations. Genome research 27:885-896.

De Bodt S., Maere S., Van de Peer Y. 2005. Genome duplication and the origin of angiosperms. Trends in ecology \& evolution 20:591-597.

Dessimoz C., Boeckmann B., Roth ACJ., Gonnet GH. 2006. Detecting non-orthology in the COGs database and other approaches grouping orthologs using genome-specific best hits. Nucleic acids research 34:3309-3316.

Dewey CN. 2011. Positional orthology: putting genomic evolutionary relationships into context. Briefings in bioinformatics 12:401-412. 
408 Glover NM., Redestig H., Dessimoz C. 2016. Homoeologs: What Are They and How Do We 409 Infer Them? Trends in plant science 21:609-621.

410 Hirulkar S., Damle M., Rathee V., Hardas B. 2014. Design of Automatic Car Breaking System 411 Using Fuzzy Logic and PID Controller. In: 2014 International Conference on Electronic 412 Systems, Signal Processing and Computing Technologies. 413-418.

413 Hu Y., Flockhart I., Vinayagam A., Bergwitz C., Berger B., Perrimon N., Mohr SE. 2011. An 414 integrative approach to ortholog prediction for disease-focused and other functional studies. $415 \quad$ BMC bioinformatics 12:357.

416 International Wheat Genome Sequencing Consortium (IWGSC). 2014. A chromosome-based 417 draft sequence of the hexaploid bread wheat (Triticum aestivum) genome. Science $418 \quad 345: 1251788$.

419 Marchler-Bauer A., Bo Y., Han L., He J., Lanczycki CJ., Lu S., Chitsaz F., Derbyshire MK., 420 Geer RC., Gonzales NR., Gwadz M., Hurwitz DI., Lu F., Marchler GH., Song JS., Thanki 421 N., Wang Z., Yamashita RA., Zhang D., Zheng C., Geer LY., Bryant SH. 2017. CDD/SPARCLE: functional classification of proteins via subfamily domain architectures. Nucleic acids research 45:D200-D203.

Ma J., Stiller J., Berkman PJ., Wei Y., Rogers J., Feuillet C., Dolezel J., Mayer KF., Eversole K., Zheng Y-L., Liu C. 2013. Sequence-based analysis of translocations and inversions in bread wheat (Triticum aestivum L.). PloS one 8:e79329.

427 Pryszcz LP., Huerta-Cepas J., Gabaldón T. 2011. MetaPhOrs: orthology and paralogy 428 predictions from multiple phylogenetic evidence using a consistency-based confidence $429 \quad$ score. Nucleic acids research 39:e32.

430 Remm M., Storm CEV., Sonnhammer ELL. 2001. Automatic clustering of orthologs and in- 
431

432

433

434

435

436

437

438

439

440

441

442

443

444

445

446

447

448

449

450

451

452

453

paralogs from pairwise species comparisons1. Journal of molecular biology 314:10411052.

Roth ACJ., Gonnet GH., Dessimoz C. 2008. Algorithm of OMA for large-scale orthology inference. BMC bioinformatics 9:518.

Smith TF., Waterman MS. 1981. Identification of common molecular subsequences. Journal of molecular biology 147:195-197.

Sutphin GL., Mahoney JM., Sheppard K., Walton DO., Korstanje R. 2016. WORMHOLE: Novel Least Diverged Ortholog Prediction through Machine Learning. PLoS computational biology 12:e1005182.

Torres A., Nieto JJ. 2006. Fuzzy logic in medicine and bioinformatics. Journal of biomedicine \& biotechnology 2006:91908.

Train C-M., Glover NM., Gonnet GH., Altenhoff AM., Dessimoz C. 2017. Orthologous Matrix (OMA) algorithm 2.0: more robust to asymmetric evolutionary rates and more scalable hierarchical orthologous group inference. Bioinformatics 33:i75-i82.

Wendel JF., Cronn RC. 2003. Polyploidy and the Evolutionary History of Cotton. Advances in Agronomy 78:139.

Zhang T., Hu Y., Jiang W., Fang L., Guan X., Chen J., Zhang J., Saski CA., Scheffler BE., Stelly DM., Hulse-Kemp AM., Wan Q., Liu B., Liu C., Wang S., Pan M., Wang Y., Wang D., Ye W., Chang L., Zhang W., Song Q., Kirkbride RC., Chen X., Dennis E., Llewellyn DJ., Peterson DG., Thaxton P., Jones DC., Wang Q., Xu X., Zhang H., Wu H., Zhou L., Mei G., Chen S., Tian Y., Xiang D., Li X., Ding J., Zuo Q., Tao L., Liu Y., Li J., Lin Y., Hui Y., Cao Z., Cai C., Zhu X., Jiang Z., Zhou B., Guo W., Li R., Chen ZJ. 2015. Sequencing of allotetraploid cotton (Gossypium hirsutum L. acc. TM-1) provides a resource for fiber 
454 improvement. Nature biotechnology 33:531-537.

455 
Table $\mathbf{1}$ (on next page)

Definitions of terms related to biology 
1

\begin{tabular}{|c|c|}
\hline Polyploidy & $\begin{array}{l}\text { Having more than two sets of homologous chromosomes; the result of } \\
\text { genome doubling. }\end{array}$ \\
\hline PAM units & $\begin{array}{l}\text { Point } A \text { ccepted } M \text { utation. A measure of evolutionary distance; the amount of } \\
\text { amino acid substitutions per } 100 \text { amino acids of a protein sequence. } 1 \text { PAM } \\
\text { unit means that } 1 \% \text { of the amino acids were replaced since the divergence of } \\
\text { the two protein sequences. }\end{array}$ \\
\hline homoeolog & $\begin{array}{l}\text { Genes of an allopolyploid which started diverging by a speciation event, and } \\
\text { were brought back to the same genome via a hybridization event. They can } \\
\text { be thought of as orthologs between subgenomes. }\end{array}$ \\
\hline allopolyploid & $\begin{array}{l}\text { A species which has more than one set of homologous chromosomes due to a } \\
\text { whole genome duplication via hybridization. }\end{array}$ \\
\hline subgenome & One of the genome sets in a polyploid. \\
\hline synteny & $\begin{array}{l}\text { The degree of gene position conservation between two diverging segments of } \\
\text { chromosomes, in this case between two homoeologous chromosomes. }\end{array}$ \\
\hline $\begin{array}{l}\text { Evolutionary } \\
\text { distance }\end{array}$ & The amount of divergence between two protein sequences. \\
\hline $\begin{array}{l}\text { Total copy } \\
\text { number }\end{array}$ & $\begin{array}{l}\text { An assessment of the amount of duplication for a given homoeolog pair. In } \\
\text { this paper, it is the sum of the homoeologs for both genes of the pair. }\end{array}$ \\
\hline
\end{tabular}

\section{Table 1: Definitions of terms related to biology}


Table 2 (on next page)

Definition of terms related to fuzzy logic 
1

\begin{tabular}{|l|l|}
\hline Fuzzy logic & $\begin{array}{l}\text { A type of mathematical logic based on natural language where truth is } \\
\text { considered on a continuous scale as degrees of truth rather than binary true } \\
\text { or false. Fuzzy logic resembles human reasoning and intuition because it } \\
\text { uses classes with unsharp boundaries, defined with natural language. }\end{array}$ \\
\hline Control system & The mathematical models which make up the fuzzy inference process. \\
\hline $\begin{array}{l}\text { Universe of } \\
\text { discourse }\end{array}$ & A set of all possible values defined for a fuzzy input or output. \\
\hline $\begin{array}{l}\text { Membership } \\
\text { function }\end{array}$ & $\begin{array}{l}\text { A function, normally visualized graphically, which denotes a fuzzy set. The } \\
\text { membership function represents the degree (between } 0 \text { and 1) to which an } \\
\text { element in the universe of discourse belongs. The membership functions } \\
\text { represent fuzzy sets also represent linguistic variables, which overlap so } \\
\text { that an input may belong to two categories, each to a certain degree. }\end{array}$ \\
\hline Fuzzification & $\begin{array}{l}\text { The process of translating a crisp input to a fuzzy one. This is the first step } \\
\text { of the fuzzy inference process, where the crisp input gets mapped to its } \\
\text { fuzzy set based on the membership functions. }\end{array}$ \\
\hline Defuzzification & $\begin{array}{l}\text { The process of converting the fuzzy output derived from the fuzzy } \\
\text { inference process to a crisp output. }\end{array}$ \\
\hline Fuzzy rules & $\begin{array}{l}\text { A set of "if ... then" rules needed for mapping the fuzzy input to the fuzzy } \\
\text { output. These rules are based on a human's expertise, knowledge, and } \\
\text { intuition. The fuzzy rules are defined and stored in the lookup table. }\end{array}$ \\
\hline $\begin{array}{l}\text { Crisp input or } \\
\text { output }\end{array}$ & $\begin{array}{l}\text { Input or output which has a quantitative value, limited to the range of the } \\
\text { universe of discourse. }\end{array}$ \\
\hline $\begin{array}{l}\text { Fuzzy inference } \\
\text { process }\end{array}$ & $\begin{array}{l}\text { The fuzzy inference process consists of taking the crisp input, fuzzifying it, } \\
\text { combining it with the fuzzy rules, and defuzzyfying, resulting in a crisp } \\
\text { output. }\end{array}$ \\
\hline $\begin{array}{l}\text { A set with unsharp boundaries, as defined by the membership function. } \\
\text { Fuzzy sets allow for its members to belong to more than one set a the same } \\
\text { time, to some partial degree. }\end{array}$ \\
\hline
\end{tabular}

2 Table 2: Definition of terms related to fuzzy logic 
Table 3 (on next page)

Universe of discourse for all variables of the fuzzy sets 
1

\begin{tabular}{|c|c|c|c|c|}
\hline Variable & Input or Output & Minimum & Maximum & Step \\
\hline distance & Input & 0 & distance max & .01 \\
\hline synteny score & Input & 0 & 1 & .01 \\
\hline total copy nr & Input & 2 & total copy nr max & 1 \\
\hline Confidence & Output & 0 & 100 & 1 \\
\hline
\end{tabular}

2 Table 3. Universe of discourse for all variables of the fuzzy sets. 


\section{Table 4 (on next page)}

Membership functions for all variables of the fuzzy sets.

Each membership class is a gaussian curve, with the central point and standard deviation defined here. 
1

\begin{tabular}{|c|c|c|c|}
\hline Variable & Membership class & Central point & Standard deviation \\
\hline Distance & Low & 0 & Distance maximum / 10 \\
\hline Distance & Med & Distance maximum / 4 & Distance maximum / 10 \\
\hline Distance & High & Distance maximum & Distance maximum / 2.5 \\
\hline Synteny & Low & 0 & 0.15 \\
\hline Synteny & Med & 0.3 & 0.15 \\
\hline Synteny & High & 1 & 0.4 \\
\hline TotalCopyNr & Low & TotalCopyNr median & TotalCopyNr median \\
\hline TotalCopyNr & Med & 4 * TotalCopyNr median & $1.5 *$ TotalCopyNr median \\
\hline TotalCopyNr & High & TotalCopyNr maximum & TotalCopyNr maximum / 2.5 \\
\hline Confidence & Very Low & 0 & 20 \\
\hline Confidence & Low & 50 & 10 \\
\hline Confidence & Med & 70 & 10 \\
\hline Confidence & High & 90 & 10 \\
\hline Confidence & Very high & 100 & 10 \\
\hline
\end{tabular}

2 Table 4. Membership functions for all variables of the fuzzy sets. Each membership class is a 3 gaussian curve, with the central point and standard deviation defined here.

4

5 


\section{Figure 1}

Method for calculating the local synteny score for a homoeolog pair

For each pair, a window of 10 genes surrounding each homoeolog was obtained. The synteny score is the mean proportion of genes in the windows that are homoeologous. A) An example of a pair with a high synteny score, and B) and example with a low synteny score. 
A

chr A01

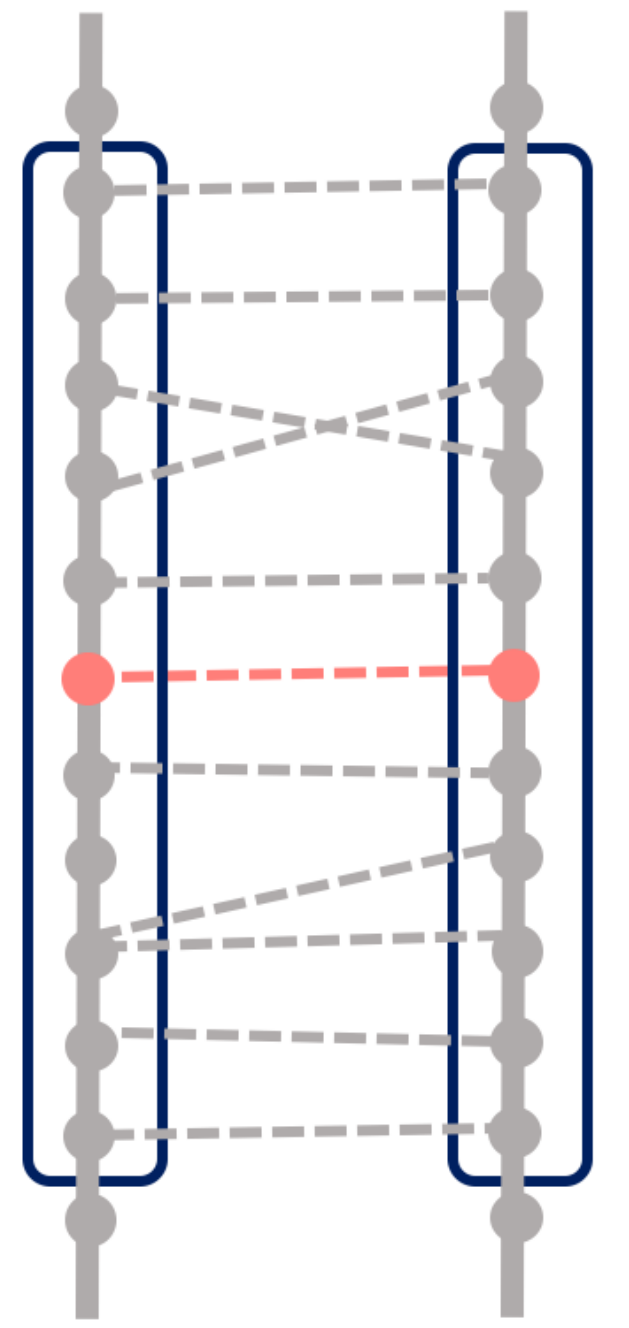

$9 / 10$

$10 / 10$

Synteny Score $=0.95$
B

chr A01

chr D05

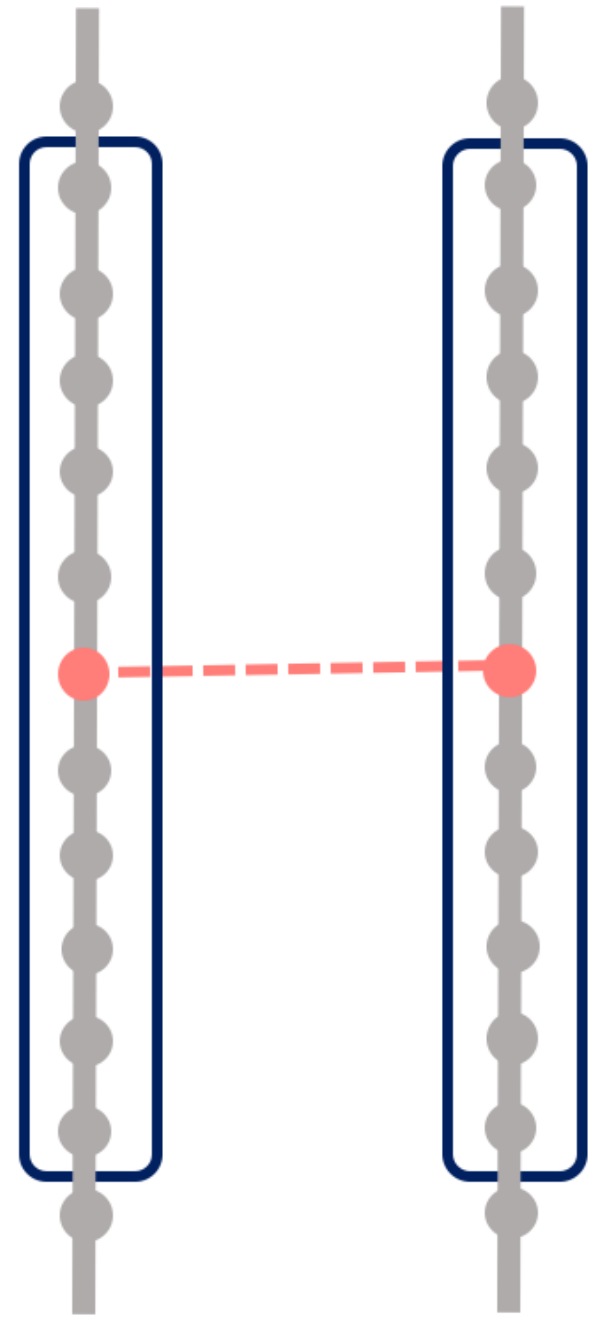

$0 / 10$

$0 / 10$

Synteny Score $=0$ 


\section{Figure 2}

Heatmaps showing the number of homoeologs predicted with OMA between each of the chromosomes in the two subgenomes in (A) GOSHI, (B) BRANA, and (C-E) WHEAT.

Homoeologs that were on scaffolds or "randoms" were mapped to their respective chromosomes. "Off-diagonal" chromosomes, i.e. different chromosome groups, with an increased frequency of homoeologs are consistent with known reciprocal translocations. 
A

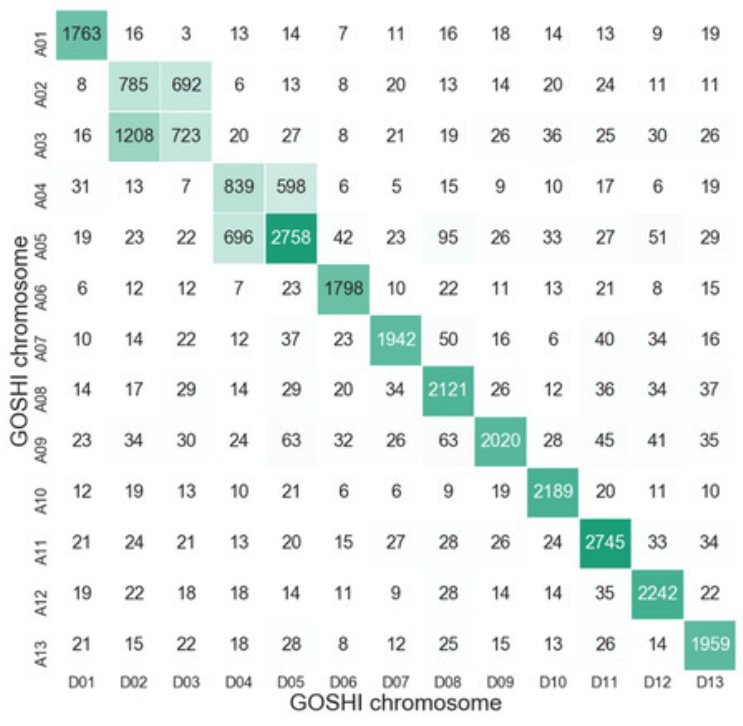

C

\section{B}

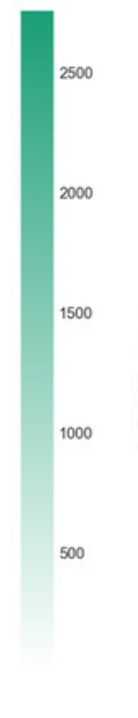

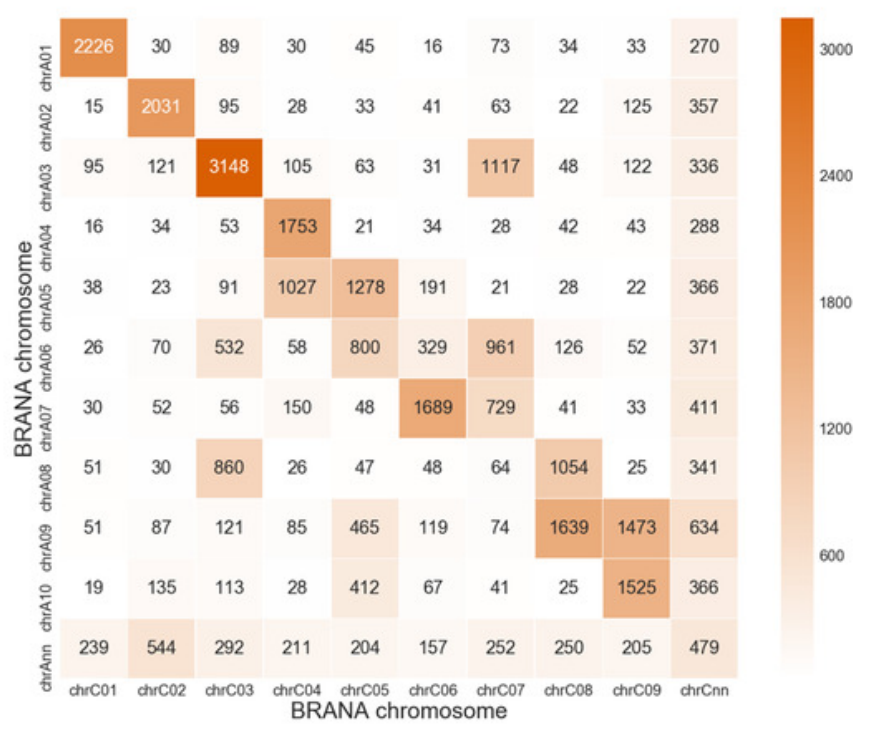

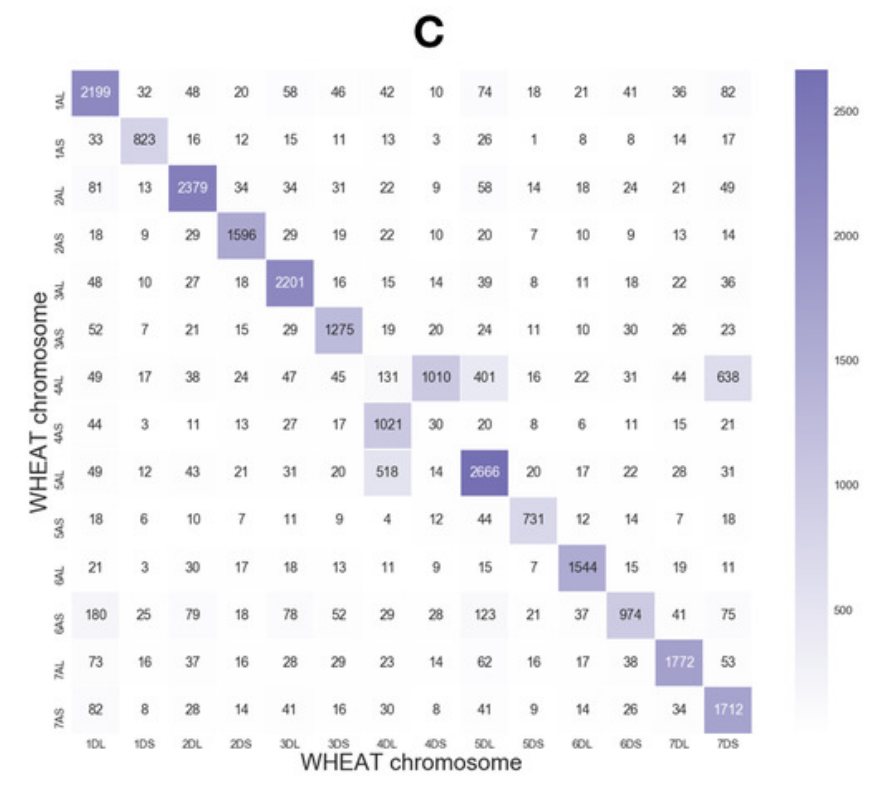

\section{E}

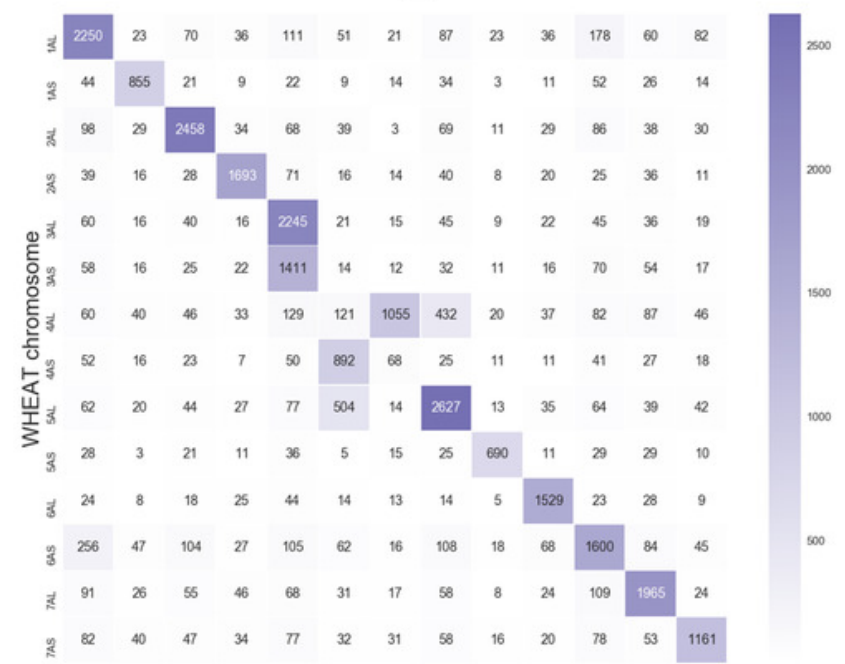


Figure 3

Schema of the known large reciprocal translocations in GOSHI

Chromosome segments were exchanged between A02 and A03, as well as A04 and A05 in subgenome A. This would result in an increased frequency of homoeolog pairs predicted between chromosomes A02/D03, A03/D02, A04/D05, and A05/D04, which we observe with the homoeolog pairs inferred by OMA. In this figure, chromosome segments of the same color between subgenome A (Figure 3A) and subgenome D (Figure 3B) are those with a high frequency of homoeolog pairs.

\section{Subgenome A}

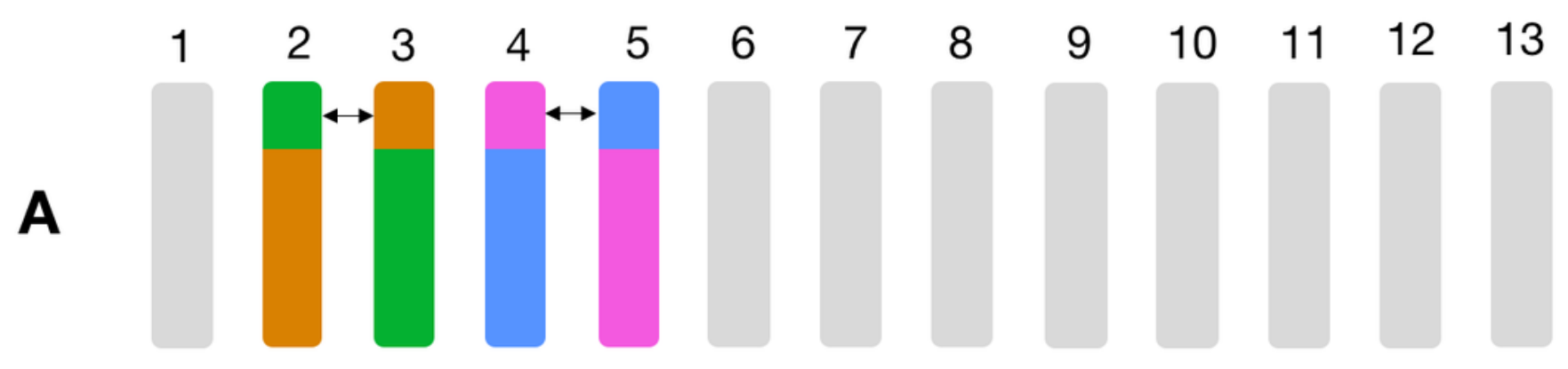

Subgenome D

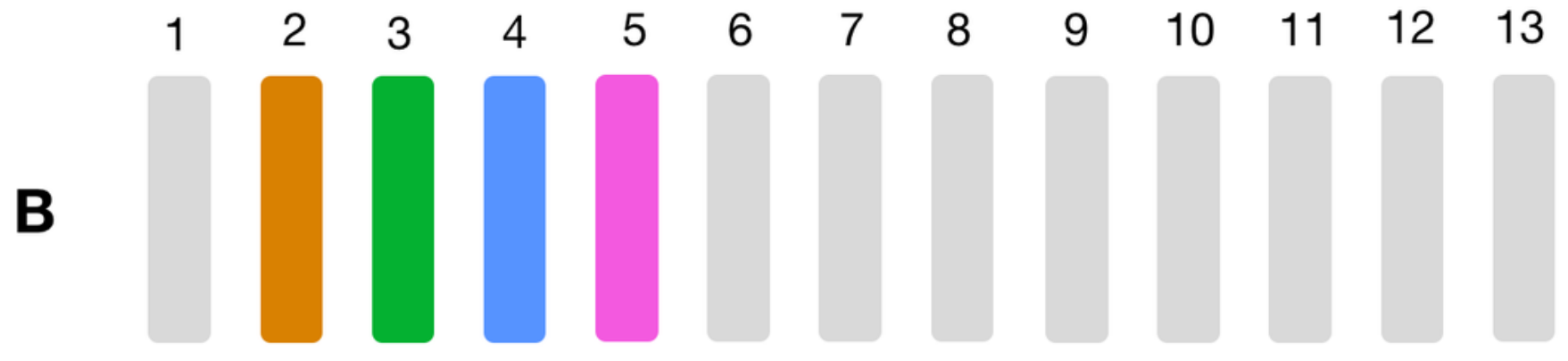


Figure 4

Homoeolog features used for fuzzy inputs

A-C) Distribution of synteny scores for pairs of homoeologs for Gossypium hirsutum, Brassica napus, and Triticum aestivum, respectively. Pairs with one or both of the homoeologs on scaffolds with only one gene on the scaffold were removed. B) Distributions of distance and C) total copy number for homoeolog pairs in each genome.
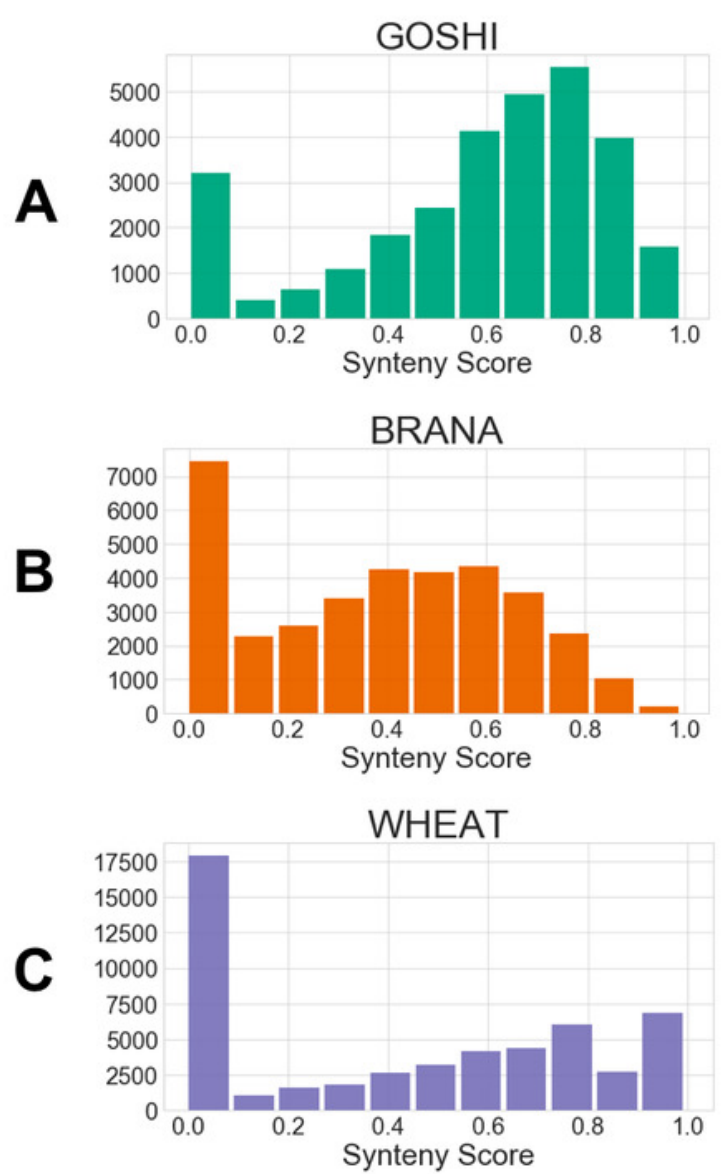

D

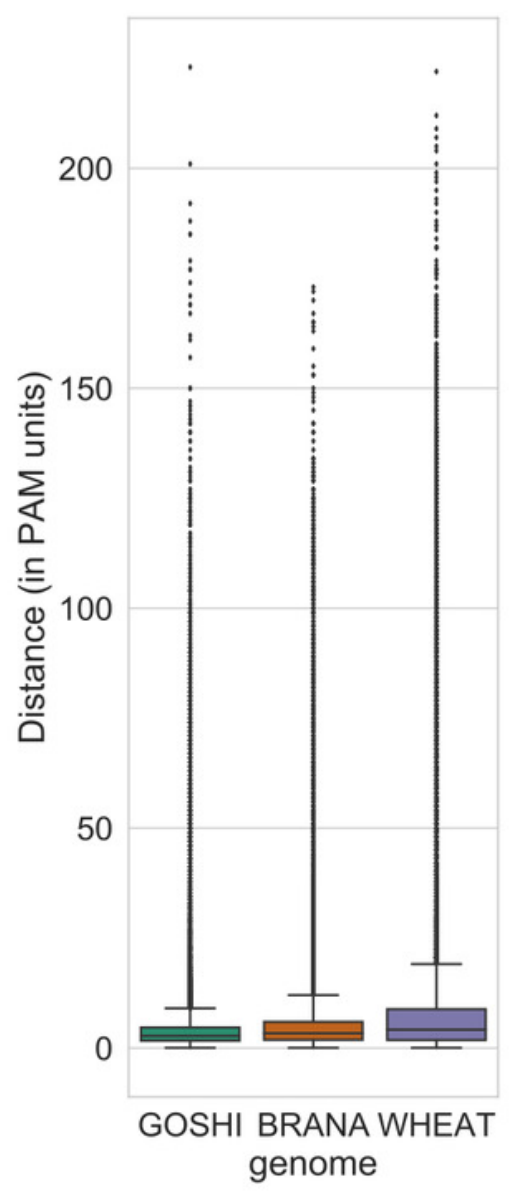

E

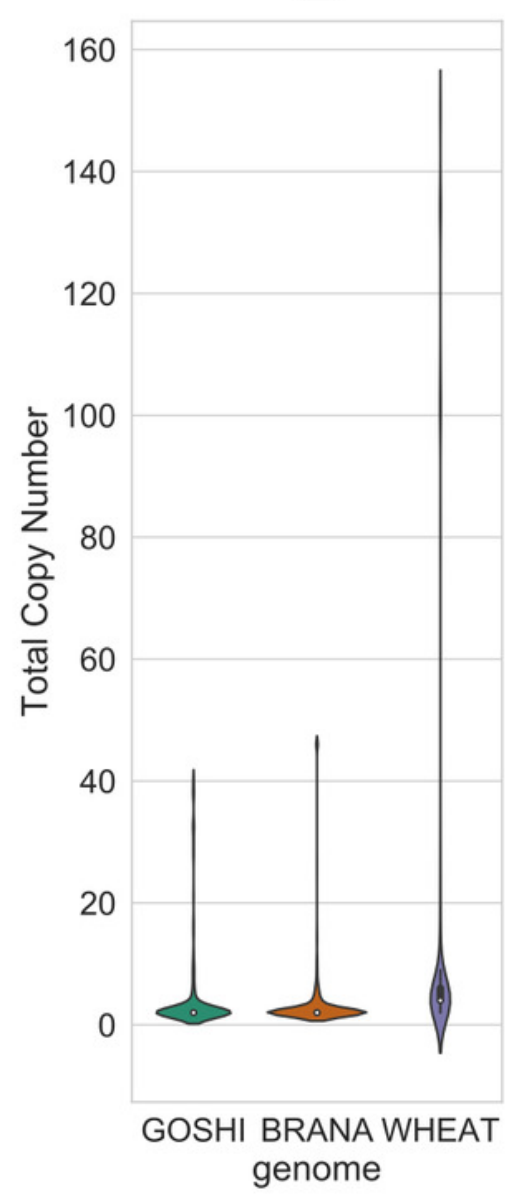


Figure 5

Membership functions for the three inputs-- synteny, distance, and total copy number-for GOSHI (A-C), BRANA (D-F), and WHEAT (G-I).
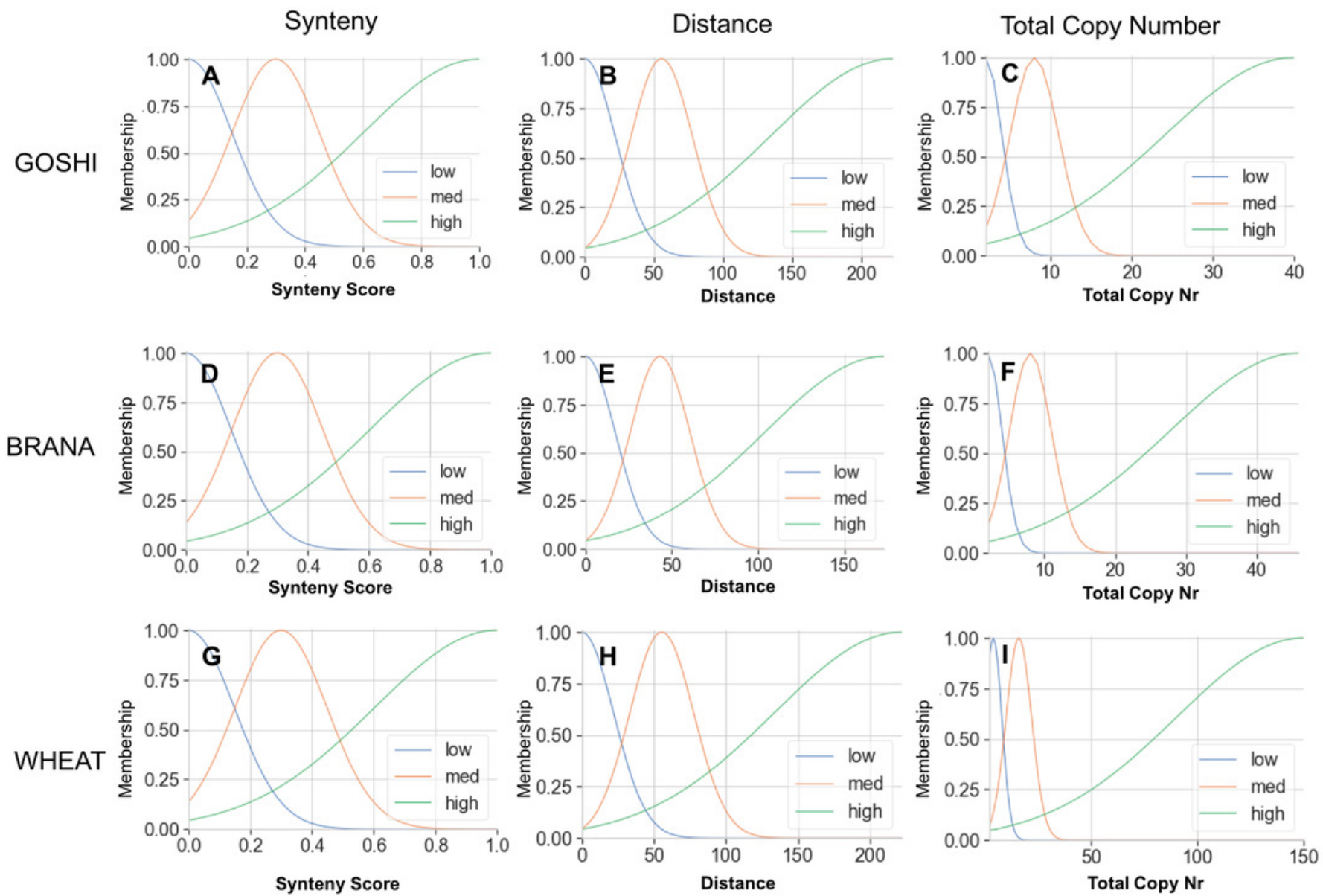
Figure 6

\author{
The Fuzzy Rules
}

A) Lookup Table. The first three columns are the inputs. The final column is the output (confidence), and reflects the same colors as the confidence membership curves in B.

A

\begin{tabular}{|c|c|c|c|}
\hline $\begin{array}{l}\text { Synteny } \\
\text { Score }\end{array}$ & Distance & $\begin{array}{c}\text { Total Copy } \\
\text { Number }\end{array}$ & Confidence \\
\hline low & high & high & very low \\
\hline low & high & low & low \\
\hline low & high & med & low \\
\hline low & med & high & low \\
\hline med & high & high & low \\
\hline high & high & high & med \\
\hline low & low & high & med \\
\hline low & med & low & med \\
\hline low & med & med & med \\
\hline med & high & low & med \\
\hline med & high & med & med \\
\hline med & med & high & med \\
\hline med & med & low & med \\
\hline med & med & med & med \\
\hline low & low & low & med \\
\hline low & low & med & med \\
\hline high & high & low & high \\
\hline high & high & med & high \\
\hline high & low & high & high \\
\hline high & low & med & high \\
\hline high & med & high & high \\
\hline high & med & low & high \\
\hline high & med & med & high \\
\hline med & low & high & high \\
\hline med & low & med & high \\
\hline med & low & low & high \\
\hline high & low & low & very high \\
\hline
\end{tabular}

B

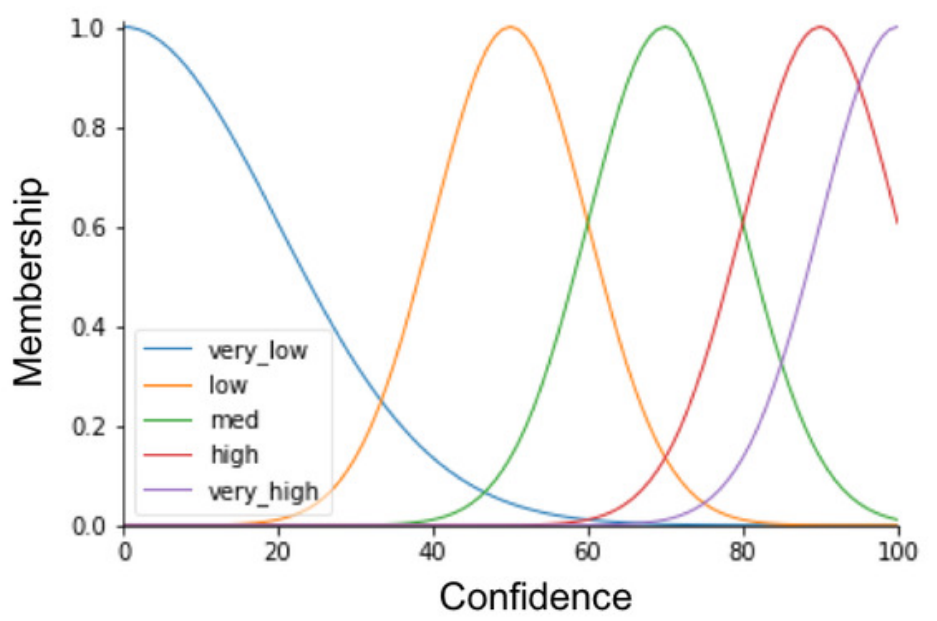


Figure 7

Distribution of crisp confidence scores after scaling to 100 for GOSHI (A), BRANA (B) and WHEAT (C).

A

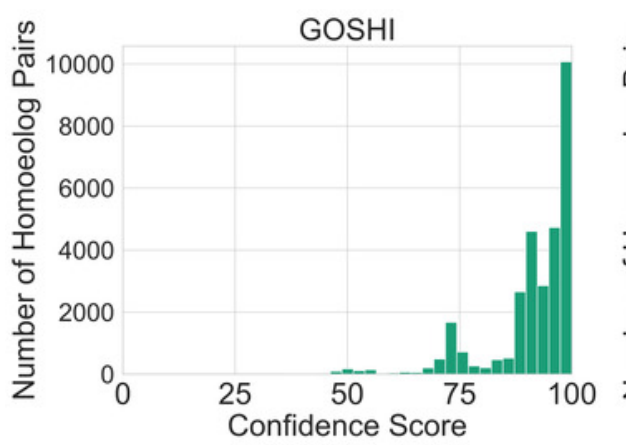

B

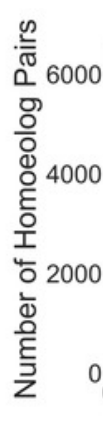

BRANA

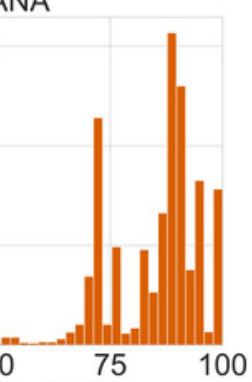

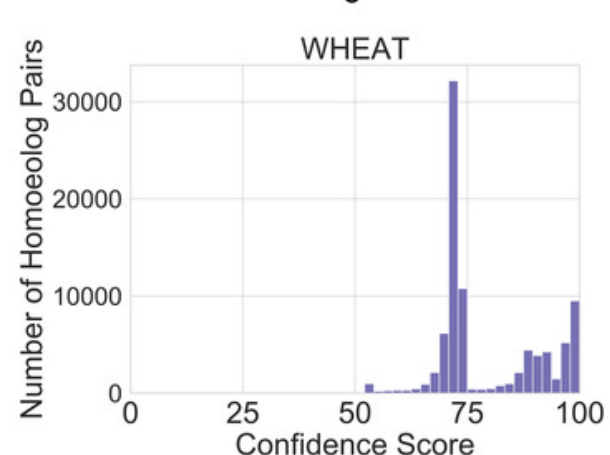


Figure 8

Distribution of the total orthology copy number, binned by confidence score, for GOSHI (A), BRANA (B) and WHEAT (C).

A

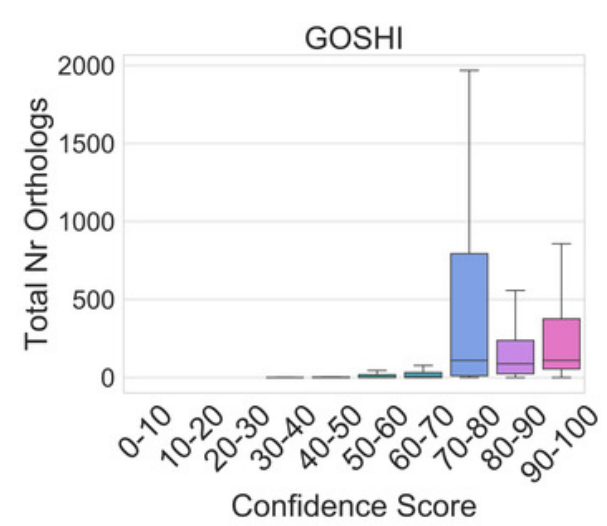

B

BRANA

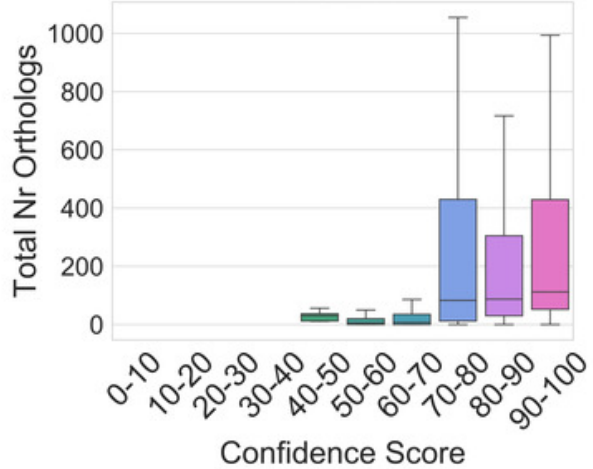

C

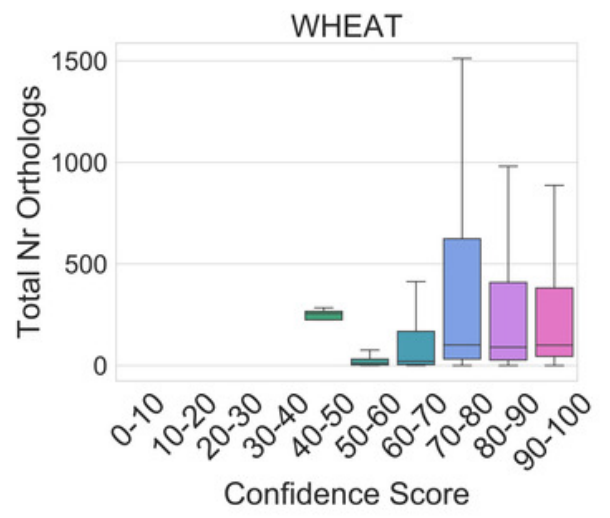


Figure 9

Comparison of new and old confidence for GOSHI (A), BRANA (B) and WHEAT (C).
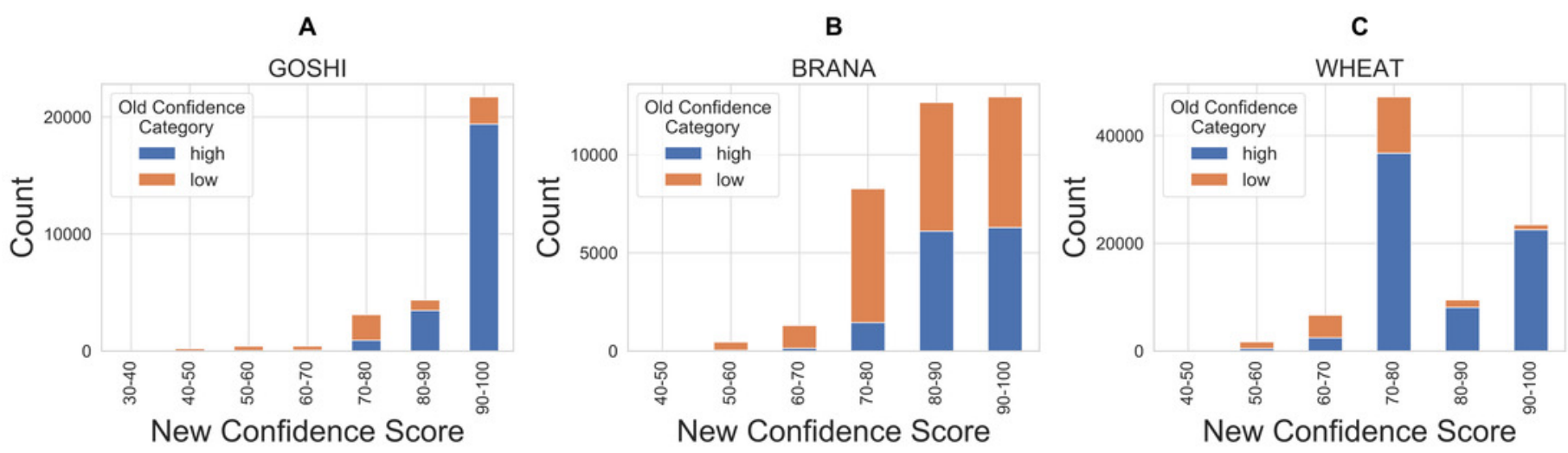